\title{
Introduction to the Special Issue of Neuropsychology Review on Cognitive Enhancement and Rehabilitation
}

\author{
Edith V. Sullivan $\cdot$ Susan F. Tapert
}

Received: 13 February 2013 / Accepted: 15 February 2013 / Published online: 27 February 2013

(C) Springer Science+Business Media New York 2013

The field of neuropsychology has accomplished much in advancing our understanding of neurobehavioral compromise; yet, guidance for rehabilitating, enhancing, compensating for, or treating discernible impairments has been far more modest. In recent years, more trials than previously conducted are evaluating the worth of cognitive rehabilitation and enhancement approaches for improvement of neuropsychological performance and day-to-day functioning, and mitigation of neuropsychiatric symptoms and disease progression. This special issue of Neuropsychology Review, "Cognitive Enhancement and Rehabilitation," features five papers, spanning applications in diseases and acquired disorders: some degenerative (i.e., Alzheimer's disease [AD] and mild cognitive impairment) and others fluctuating (i.e., alcohol use disorders and HIV infection). Future issues of the journal will focus on acquired head injury and consider rehabilitation efforts.

\section{Thoughts on Rehabilitation from Kurt Goldstein's The Organism}

As an historical introduction and context for the reviews in this issue, we have reprinted two excerpts from Kurt Goldstein's classic book, The Organism, which he dictated in 1934 (cf., forward by Oliver Sacks in the 1995 edition of The Organism). Goldstein's contributions to neuropsychology stem from his experiences as a neurologist- perhaps

E. V. Sullivan $(\bowtie)$

Department of Psychiatry and Behavioral Sciences, Stanford University School of Medicine, (MC5723), 401 Quarry Road, Stanford, CA 94305-5723, USA

e-mail: edie@stanford.edu

S. F. Tapert

Department of Psychiatry and Behavioral Sciences, University of California San Diego, School of Medicine, San Diego, CA, USA today considered a behavioral neurologist - and psychiatrist and through his studies of soldiers who had sustained head injuries in the Great World War (e.g., Aftereffects of brain injuries in war: their evaluation and treatment; the application of psychologic methods in the clinic 1942). His observations expanded the purview of neuropsychology from Broca's and Wernicke's classical studies of language to other considerations of localization and dissociation of functions, for example, in visual perception and agnosia. Goldstein also contributed substantially to quantitative neuropsychological methods by devising tests with scoring procedures aimed at defining and isolating deficits and localizing brain lesion substrates.

Of particular relevance to the current issue of Neuropsychology Review is Goldstein's philosophy of brain damage. In short, regardless of and in addition to its localization and selectivity of resulting dysfunction, Goldstein's position was that brain injury affects the organism as a whole, thereafter rendering the individual forever different from the pre-injury state. This Gestaltist view implies that recovery can never be complete. Further to this point, Goldstein declared, "there is no such thing as compensation" (page 331). Taking such a bold statement at face value could extinguish any hope of improvement-no less full recovery - by the patient or family of the afflicted, and pursuit of rehabilitation efforts by medical staff would be worthless. Putting this statement into the organism's context as a whole, however, lends a more positive interpretation and leaves room for the possibility of "recuperation," even if limited: "Rather it [health] is restored if such a relation between preserved and disturbed performances is reached" (page 331); that is, a "new normal" must be achieved to enjoy restitution of function. Thus, the affected organism seeks a "new normal" and tends to avoid uncomfortable situations, which could result in what Goldstein named a "catastrophic reaction" that could lead to a "substitute performance" as a "protective mechanism." To the extent that 
this conceptualization of adaptation to the deficit state is accurate, rehabilitation specialists would be constantly resisted by the patient, who post-injury, is biased toward a new comfort zone and disinclined to face the deficit. In his treatise on neuropsychological rehabilitation, George Prigatano presented 13 principles, the first being highly representative of Goldstein's conception of the catastrophic reaction to the sequelae of the newly incurred brain injury: "Principle 1: The clinician must begin with patient's subjective or phenomenological experience to reduce their frustrations and confusion in order to engage them in the rehabilitation process" (Prigatano 1999, page 3).

The Goldstein depiction of compensation cast a long shadow of doubt on the worth of rehabilitation to alleviate neurological disorders and even raised questions about whether true improvement ever transpires. With this historical burden of apparent negative bias, neuropsychology often eschewed work in cognitive rehabilitation and more safely focused on description of the disordered brain and mind. Furthered by current quantitative approaches from neuroimaging and neuropathology, refined description and neural mechanisms of these functional impairments have been forthcoming. But as fundamentally relevant to neuroscience as are neuropsychological descriptions of patterns of sparing and impairment, it may now be time to pursue with the same vigor and rigor as applied to description and discovery of mechanism the next frontier in neuropsychology - development of methods to build on the brain's potential for functional reorganization in efforts to "recover" from insult and to determine the scope, limits, and maintenance of recovery. For inspiration, we might start with readings by pioneers in the area, such as Ben-Yishay, Luria, Christensen, Prigatano, Weinstein, and Goldstein (cf., Prigatano and Weinstein 1996).

Promising exemplars of recovery have been forthcoming even in the current news, such as the remarkable progress in functioning made from a near fatal gunshot wound to the head of United States Congresswoman, Gabrielle Giffords. Assuming cause and effect, 2 years of intensive rehabilitation has resulted in substantial restitution in speech, mobility, and emotion; success of these efforts was likely enhanced by the exceptional family support, medical care, rehabilitation options, and strong constitution and determination defining Congresswoman Giffords' premorbid personality. Echoing Goldstein regarding the role of the premorbid personality in coping with the new normal is Prigatano's second principle: "The patient's symptom picture is a mixture of premorbid cognitive and personality characteristics as well as neuropsychological changes directly associated with brain pathology" (Prigatano 1999, page 3).

\section{In This Issue}

We invite you to start with the excerpts from Kurt Goldstein and then jump the better part of a century to the present with our initial review of neuropsychological rehabilitation in this millennium.

In the first of five new papers in this issue, "Crosswords to computers: A critical review of popular approaches for cognitive enhancement," Jak and colleagues review empirical evaluations of electronic cognitive training programs across conditions, including brain injury, brain tumor, stroke, severe mental illness, and enhancement of function in healthy individuals. These approaches, often video games, commonly result in improvements on the training tasks, although lasting generalizability to other performances and everyday functions is less clear. A useful table lists the growing array of cognitive enhancement options that have been objectively evaluated.

Next, in their paper "A role for cognitive rehabilitation in increasing the effectiveness of treatment for alcohol use disorders," Bates and colleagues discuss a recent resurgence of interest in approaches that promote cognitive recovery in alcohol dependent individuals. These efforts have achieved some success in improving and maintaining treatment outcome. This review lends a hopeful update to the September 2007 issue of this journal on cognitive impairments associated with alcohol dependence.

The review by Choi and Twamley, "Cognitive enhancement therapies for Alzheimer's disease: A review of methods to improve treatment engagement and selfefficacy," covers cognitive rehabilitation therapies and related adherence issues relevant to AD. Despite the inexorable decline to total functional dependence and premature death marking $\mathrm{AD}$, this paper delineates approaches for maximizing the benefit of these treatments and emphasizes the need to engage and motivate patients - and caregiversuntil a therapeutic "dose" is achieved.

"Efficacy of cognitive rehabilitation therapies for mild cognitive impairment (MCI) in older adults: Working toward a theoretical model and evidence-based interventions" presents an evaluation of the efficacy of 14 randomized controlled trials of cognitive rehabilitation therapies for MCI. As a synthesis of these studies, Huckans et al. propose a model with four intervention targets: cognitive compromise, functional compromise, neuropsychiatric symptoms, and progression to dementia.

The final paper, "Cognitive neurorehabilitation of HIVassociated neurocognitive disorders: A qualitative review and call to action," summarizes and critiques the emerging literature on the efficacy of cognitive rehabilitation for remediating HIV-associated neurocognitive impairment. Weber et al. propose a decision-tree for diagnostic criteria and a conceptual model depicting possible targets for prevention and rehabilitation of the real-world neurocognitive deficits of HIV.

With a central mission of the United States National Institutes of Health for translational medicine together with 
the prolongation of life using improved care and medications, notable in HIV infection, cognitive rehabilitation and enhancement strategies have gained new popularity to improve performance in neurological and neuropsychiatric conditions, which, by definition, affect function. Until recently, the cognitive rehabilitation field has been characterized by a paucity of research with adequate control groups, sufficiently long follow-ups, and meaningful outcome assessments to determine the extent to which training approaches improve cognition, day-to-day functionality, and quality of life if these functional areas are modified by intervention. Surely, more rigorous study is required to devise effective therapeutic methods and to evaluate their worth with objectivity and to capitalize on the brain's potential for functional reorganization promoting useful performance and diminishing impairment, even if short of full recovery. We hope that this issue of the journal will encourage interest in the many facets and challenges facing neuropsychological rehabilitation, from creation of methods for evaluation to understanding how revision of functional neural connections might occur during the rehabilitation process, with the common goal to improve the lives of brain injured patients.

Acknowledgments AA017168 (E.V.S.)

\section{References}

Goldstein, K. (1942). Aftereffects of brain injuries in war. New York: Grune and Stratton.

Goldstein, K. (1995). The organism. New York: Zone Books.

Prigatano, G. P. (1999). Principles of neuropsychological rehabilitation. New York: Oxford University Press.

Prigatano, G. P., \& Weinstein, E. A. (1996). Neuropsychological rehabilitation, 6, 305-326. 